\title{
Effect of Mg contents on Trapping Efficiency in Al-Mg Alloy Using Positron Annihilation Spectroscopy
}

\author{
N. A. Kamel \\ Faculty of Science, Physics Dept., El-Minia University, Minia, Egypt. \\ e-mail: nasser_ak_62@yahoo.com
}

Positron lifetime technique and Doppler broadening S- parameter are used for Al-Mg alloy investigation, namely 5005, 5051, 5052 and 5083. The trapping efficiency was estimated for the mentioned alloys as $2.42 \times 10^{9}$, $2.29 \times 10^{9}, 2.24 \times 10^{9}$ and $2.27 \times 10^{9} \mathrm{~s}^{-1} \mathrm{~cm}^{3}$ respectively and the trapping crosssection was estimated to be $2.66 \times 10^{-16}, 2.14 \times 10^{-16}, 2.10 \times 10^{-16}$ and $3.51 \times 10^{-16}$ $\mathrm{cm}^{2}$ respectively. It is strongly clear that, the mean lifetime and S-parameter have the same behaviour as a function of deformation degree and saturates at the same value of thickness reduction.

\section{Introduction:}

The present work aims to investigate the dependence of trapping efficiency and trapping cross-section of the Mg content atoms in a series of commercial Al-alloys by positron annihilation spectroscopy. These parameters can be used to estimate the dissslocation density and concentration of defect which reveal the state of the material. Plastic deformation produces various types of defects like dislocations and vacancies both with high concentrations. Depending on the degree of deformation and crystal orientation the interactions between dislocations causes the formation of bundles, cell or wall structures leading to a modulation of the dislocations density by a factor of $10^{2}$ on a typical length scale of $1 \mu \mathrm{m}$ [1].

\section{Experimental Procedure:}

Samples were cleaned by electropolishing in a solution of $75 \%$ of methanol and 25\% nitric acid[2] by (Lectrupol-5) struers. All samples are rinsed in pure acetone and rinsed in distilled water and dried. Samples are annealed for $8 \mathrm{~h}$ at $773 \mathrm{~K}$, then plastically deformed at room temperature using a hydraulic press to obtain a certain thickness reduction from the original. The deformation of the samples is expressed in percent use (\%) of the thickness. The 
positron lifetime was recorded by a time spectrometer using a fast/fast coincidence method. The time resolution of the system using a ${ }^{60} \mathrm{Co}$ source, approximately 280 ps (FWHM). Each spectrum was accumulated for a period of 1 hour. Positron lifetimes were measured at RT in 12 pairs of deformed Al-Mg alloys, namely 5005, 5051, 5052 and 5083, which were prepared with wellknown thickness $\left(0.4 \times 1.5 \times 1.5 \mathrm{~cm}^{3}\right)$. The samples were annealed for $15 \mathrm{~h}$ at a temperature of $823 \mathrm{~K}$. The samples were deformed at RT to different degrees of thickness reduction from $1.5 \%$ up to $47 \%$. Figure (1) shows the positron lifetime spectra for sample 5051 with $0 \%$ and $30 \%$ deformation. In this figure it can be seen, that the variation on the right side of the spectrum is thickness reduction dependent (trapping of positrons).

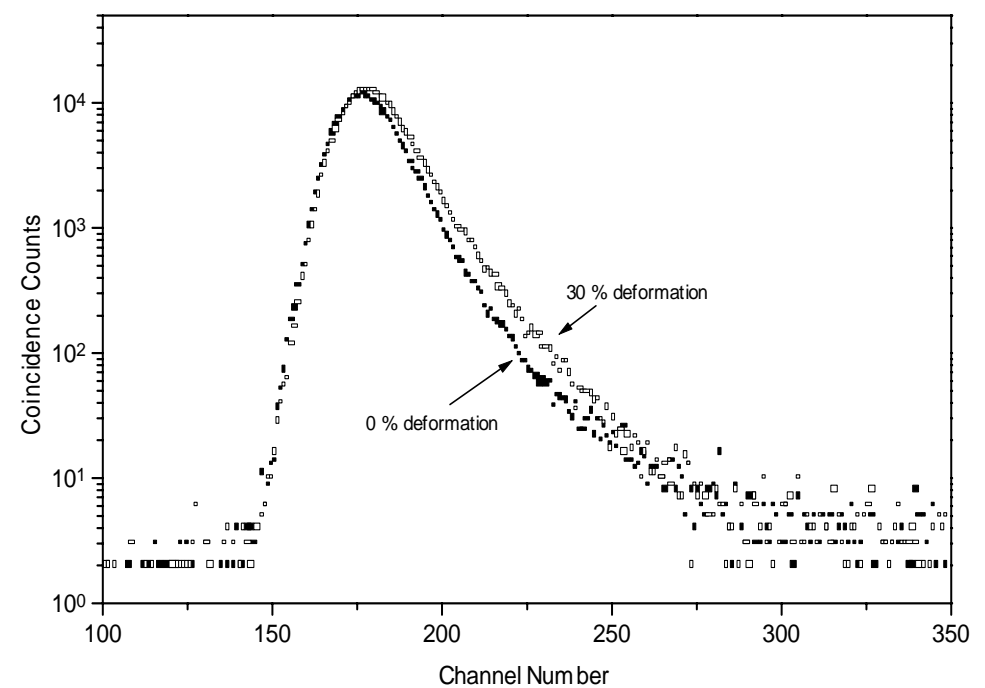

Fig. (1): Positron life time spectra for Al-Mg alloy with $0 \%$ and $0 \%$ deformation.

\section{Results and Discussion:}

Figures (2-5) represent the value of the mean lifetime $\tau$ as a function of the thickness reduction in percentage (\%). It is clear that the mean lifetime $\tau$ increases as a function of the thickness reduction (Deformation ratio) up to a certain value $10 \%$ approximately where the mean lifetime is approximately constant for each sample as indicated in Table (1). This constant value represents saturation trapping of positrons in defects. The results were interpreted using Baram and Rosen's results [3], which are as follows. A dislocation is considered to be as a chain of spherical scattering centers of density $\rho$, given by 


$$
\rho^{\prime}\left(\mathrm{cm}^{-3}\right)=\frac{\rho\left(\mathrm{cm}^{-2}\right)}{b}
$$

where $\mathrm{b}$ is the Burger's vector. The trapping probability per second, $\kappa_{\mathrm{t}}$, is proportional to dislocation concentration as:

$$
\kappa_{t}=v \rho
$$

where $v\left(\mathrm{~s}^{-1} \mathrm{~cm}^{3}\right)$ is the trapping efficiency, which is expressed in terms of the collision cross section $\sigma$ of a free positron with a trapping center:

$$
v=\sigma \mathrm{v}
$$

where $\mathrm{v}$ is the mean thermal velocity of the thermalized positron and from the Maxwellian distribution one obtains:

$$
\begin{aligned}
& \mathrm{v}=\sqrt{\frac{8 \mathrm{~K} \mathrm{в} T}{\pi m}} \\
& \sigma=\pi \mathrm{r}_{\mathrm{o}}^{2}
\end{aligned}
$$

where $K_{B}, T$ and $m$ are the Boltzmann constant, temperature, and mass of the positron. The trapping cross section can also be described by the trapping radius (capture radius), $\mathrm{r}_{\mathrm{o}}$ as follows:

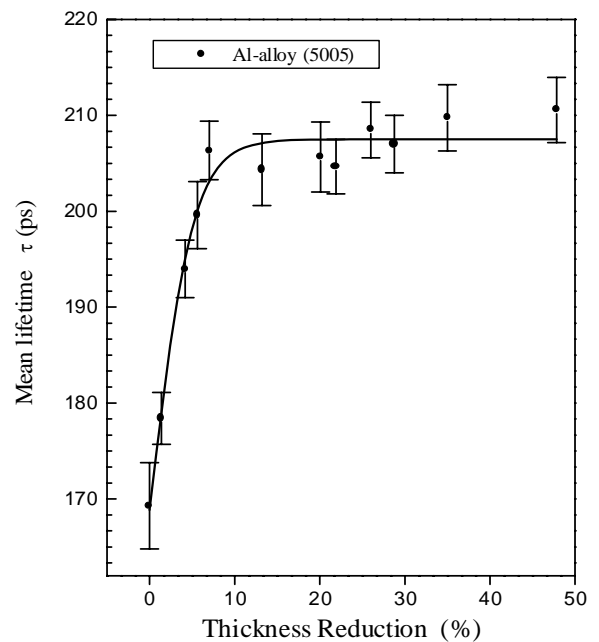

Figure 2. The mean lifetime as a function of thickness reduction (\%) for $5005 \mathrm{Al}-\mathrm{Mg}$ alloy.

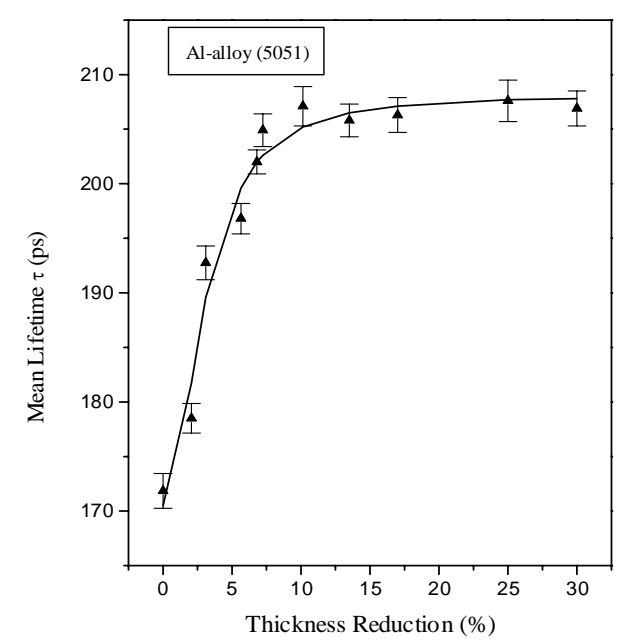

Figure 3. The mean lifetime as a function of thickness reduction (\%) for 5051 Al-Mg alloy. 


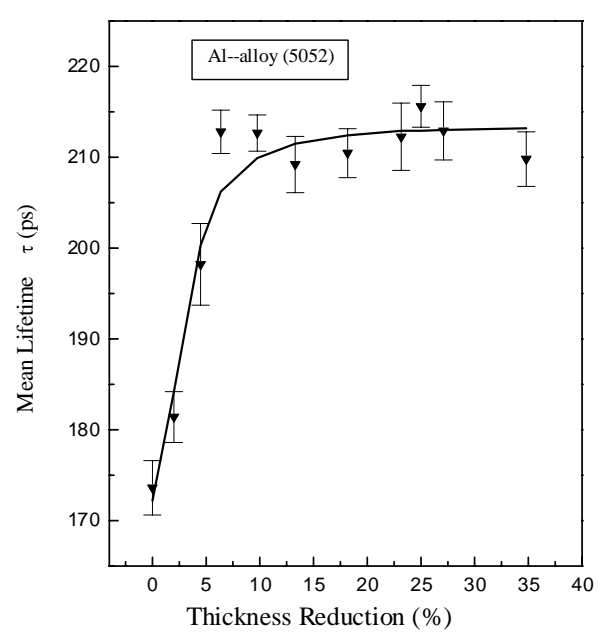

Figure 4. The mean lifetimeas a function of thickness reduction (\%) for $5052 \mathrm{Al}-\mathrm{Mg}$ alloy.

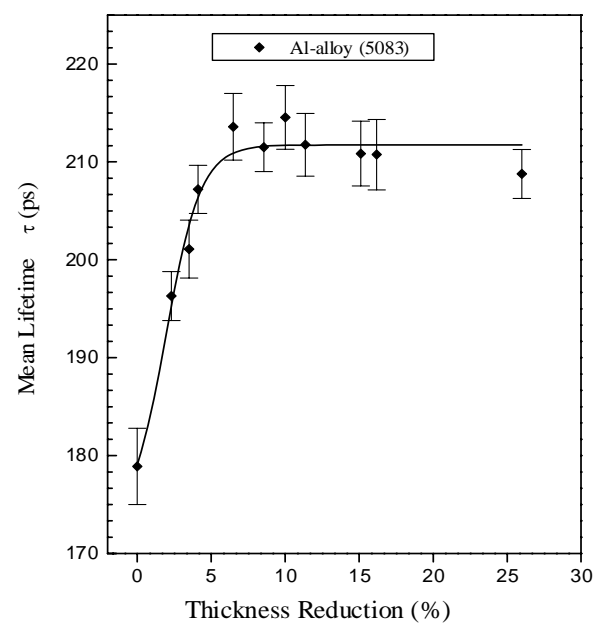

Figure 5. The mean lifetime as a function of thickness reduction (\%) for $5083 \mathrm{Al}-\mathrm{Mg}$ alloy.

The dislocation density was calculated from the relationship between dislocation density and the shear strain by Baram and Rosen's[3]. From this relationship, an expression for $\kappa_{t}$ is derived

$$
\kappa_{t}=(1.248) \cdot 10^{-3} \cdot[\log (1-R)]^{2} \cdot \frac{\sigma \mathrm{v}}{b^{3}}
$$

where $R$ is the fractional thickness reduction. Equation (6) is then substituted into the formula for positron lifetime derived by Connors and West [4]:

$$
\tau=\tau_{b}\left[\frac{1+\kappa_{t} \tau_{t}}{1+\kappa_{t} \tau_{b}}\right]
$$

where $\tau_{\mathrm{b}}$ is the lifetime for an annealed specimen and $\tau_{\mathrm{t}}$ is the lifetime for a dislocation saturated sample. The best fit of Eqn. (7) to the experimental data is shown in Figs. (2-5) by solid line. The value obtained for $\Delta \tau$, which is the increase in the positron lifetime in the limit of saturated trapping at dislocations over annihilation in the annealed samples, is approximately $47 \pm 3$ ps. The values for the lifetimes $\tau_{\mathrm{b}}$ and $\tau_{\mathrm{t}}$ obtained from the best fit, the estimated trapping efficiency $v$ and trapping cross section $\sigma$ are given in Table (1). Previous measurements for trapping efficiency and cross section for some other materials are shown in references [5-7]. 
Table (1): The values for the lifetimes $\tau_{\mathrm{b}}$ and $\tau_{\mathrm{t}}$, trapping efficiency $v$ and trapping cross section $\sigma$.

\begin{tabular}{|c|c|c|c|c|}
\hline Alloy & $\tau_{b}$ (ps) & $\tau_{t}$ (ps) & $\begin{array}{c}v \times 10^{-9} \\
\left(\mathrm{~s}^{-1} \mathrm{~cm}^{3}\right)\end{array}$ & $\begin{array}{c}\sigma \times 10^{-16} \\
\left(\mathrm{~cm}^{2}\right)\end{array}$ \\
\hline 5005 & $170.3 \pm 2.0$ & $208 \pm 2.0$ & 2.42 & 2.66 \\
5051 & $171.0 \pm 1.7$ & $209 \pm 1.9$ & 2.29 & 2.14 \\
5052 & $172.2 \pm 1.3$ & $213 \pm 2.2$ & 2.24 & 2.10 \\
5083 & $178.7 \pm 2.2$ & $212.7 \pm 2.4$ & 3.27 & 3.51 \\
\hline
\end{tabular}

Two parameters S (for central), and W (for wings) are usually used to characterize the annihilation peak. The S-parameter is more sensitive to the annihilation with low momentum valence and unbound electrons. The S-parameter defined as the ratio of the integration over the central part of the annihilation line to the total integration. The experimental peaks are analyzed through common fitting procedures, which result in parameters like the centre of gravity and the width of the distribution. The $\mathrm{W}$-parameter is more sensitive to the annihilation with high momentum core electrons and is defined as the ratio of counts in the wing regions of the peak to the total counts in the peak..

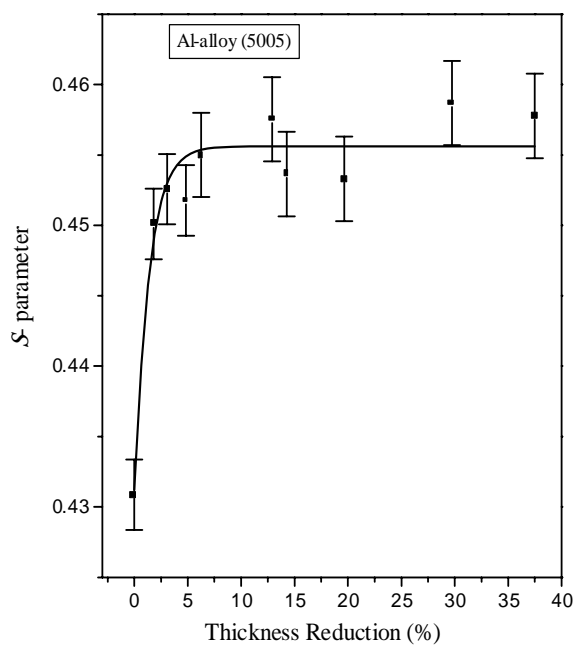

Figure 6. The S-parameter as a function of thickness reduction (\%) for $5005 \mathrm{Al}-\mathrm{Mg}$ alloy.

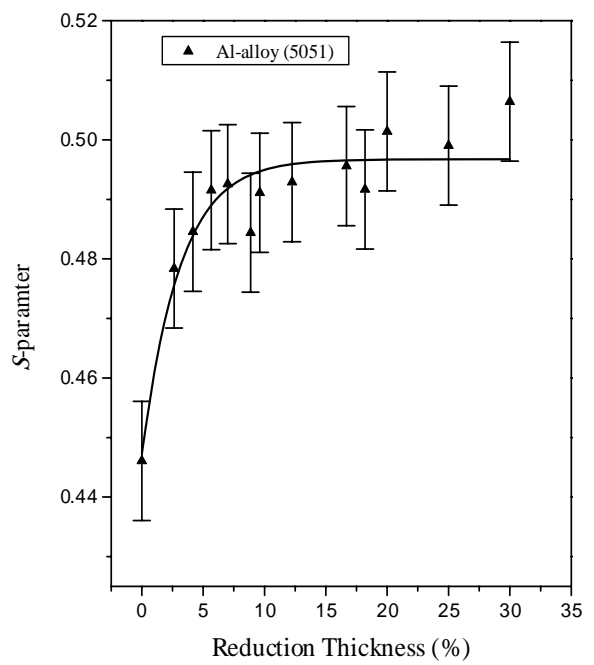

Figure 7 . The S-parameter as a function of thickness reduction (\%) for $5051 \mathrm{Al}-\mathrm{Mg}$ alloy. 
Figures (6-8) represent the S-parameter as a function of the thickness reduction for alloys 5005, 5051 and 5083, which indicate that the S-parameter has the same behavior of the lifetime measurements and the saturation of the positron trapping in defects occur at approximately the same values of the thickness reduction $10 \%$ as shown before in Figs. (2-5).

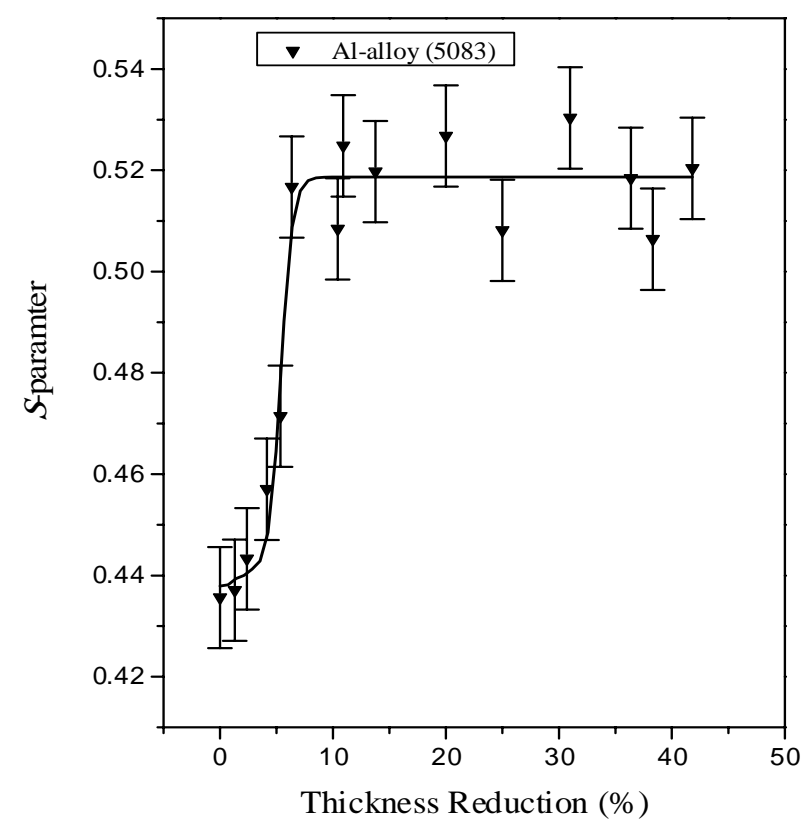

Figure 8. The S-parameter as a function of thickness reduction (\%) for $5083 \mathrm{Al}-\mathrm{Mg}$ alloy.

\section{Conclusion:}

Measurements of positron mean lifetime $\tau$ as a function of thickness reduction have been performed on a series of Al-Mg alloys. The trapping efficiency was estimated for the previously mentioned alloys as $2.42 \times 10^{9}$, $2.29 \times 10^{9}, 2.24 \times 10^{9}$ and $2.27 \times 10^{9} \mathrm{~s}^{-1} \mathrm{~cm}^{3}$ respectively and the trapping crosssection was estimated as $2.66 \times 10^{-16}, 2.14 \times 10^{-16}, 2.10 \times 10^{-16}$ and $3.51 \times 10^{-16} \mathrm{~cm}^{2}$, respectively. The S-parameter was studied as a function of thickness reduction for Al-Mg alloys. The results indicated that the S-parameter has the same behavior of $\tau$ measurements and the saturation of positron trapping $10 \%$ occurs at approximately $10 \%$ thickness reduction. This saturation due to all the positrons is trapped. 


\section{References:}

1. J. Baram and M. Rosen: phys. stat. sol., 12, 707 (1965).

2. K. C. Thompson, and J. W. Edington, "Electron Microscope specimen preparation techniques in Materials science", Conserua and Fiorini (1973).

3. J. Baram and M. Rosen: phys. stat. sol., A16, 263(1973).

4. D. C. Connors and R. N. West: Phys. Lett., 30A, 24 (1969).

5. M. A. Abdel-Rahman and E. Badawi: Jpn. J. Appl. Phys., 35. (1996).

6. E. Badawi: Egypt J. Solids, 22 (2), (1999).

7. E. Badawi, M. A. Abdel-Rahman and N. Z. El-Syed, Aluminum Transaction, 2 (1), (2000). 